\title{
Tunneling Glashow-Weinberg-Salam Model Particles from Black Hole Solutions in Rastall Theory
}

\author{
Ali Övgün $\left(\mathbb{D},{ }^{1,2}\right.$ Wajiha Javed, ${ }^{3}$ and Riasat $\mathbf{A l i}^{3}$ \\ ${ }^{1}$ Instituto de Física, Pontificia Universidad Católica de Valparaíso, Casilla 4950, Valparaíso, Chile \\ ${ }^{2}$ Physics Department, Arts and Sciences Faculty, Eastern Mediterranean University, Famagusta, Northern Cyprus, Mersin 10, Turkey \\ ${ }^{3}$ Division of Science and Technology, University of Education, Township Campus, Lahore, Pakistan \\ Correspondence should be addressed to Ali Övgün; ali.ovgun@pucv.cl
}

Received 17 April 2018; Revised 25 June 2018; Accepted 10 July 2018; Published 18 July 2018

Academic Editor: Saibal Ray

Copyright ( 2018 Ali Övgün et al. This is an open access article distributed under the Creative Commons Attribution License, which permits unrestricted use, distribution, and reproduction in any medium, provided the original work is properly cited. The publication of this article was funded by SCOAP S $^{3}$

\begin{abstract}
Using the semiclassical WKB approximation and Hamilton-Jacobi method, we solve an equation of motion for the GlashowWeinberg-Salam model, which is important for understanding the unified gauge-theory of weak and electromagnetic interactions. We calculate the tunneling rate of the massive charged W-bosons in a background of electromagnetic field to investigate the Hawking temperature of black holes surrounded by perfect fluid in Rastall theory. Then, we study the quantum gravity effects on the generalized Proca equation with generalized uncertainty principle (GUP) on this background. We show that quantum gravity effects leave the remnants on the Hawking temperature and the Hawking radiation becomes nonthermal.
\end{abstract}

\section{Introduction}

General relativity is analogously linked to the thermodynamics and quantum effects which strongly support it [1$3]$. Black holes are the strangest objects in the Universe and they arise in general relativity, a classical theory of gravity, but it is needed to include quantum effects to understand the nature of the black holes properly. After Bekenstein found a relation between the surface area and entropy of a black hole [4], Hawking theoretically showed that black holes with the surface gravity $\kappa$ radiate at temperature $\kappa / 2 \pi$ [5-7]. On the other hand, Bekenstein-Hawking radiation causes the information loss paradox because of the thermal evaporation. To solve the information paradox, recently soft-hair idea has been proposed by Hawking et al. [8].

Since Bekenstein and Hawking great contribution to the black hole's thermodynamics, the radiation from the black hole gets attention from researchers. There are many different methods to obtain the Bekenstein-Hawking radiation using the quantum field theory or the semiclassical methods. The quantum tunneling method is one of them [916]. Nozari and Mehdipour [17] have studied the Hawking radiation as tunneling phenomenon for Schwarzschild $\mathrm{BH}$ in noncommutative space-time. Nozari and Saghafi [18] have investigated the tunneling of massless particles for Schwarzschild $\mathrm{BH}$ by considering quantum gravity effects. The semiclassical tunneling method by using the HamiltonJacobi ansatz with WKB approximation is another way to obtain the Bekenstein-Hawking temperature and the tunneling rate as $\Gamma \approx \exp [-2 \operatorname{ImS}]$ [19]. Different kinds of particles such as bosons, fermions, and vector particles are used to study the tunneling of the particles from the black holes and wormholes and obtain their Hawking temperature [20-46]. Nozari and Sefidgar [47] have discussed quantum corrections approach to study $\mathrm{BH}$ thermodynamics. Nozari and Etemadi [48] have investigated the KMM seminal work in case of a maximal test particles momentum. They showed that, in the presence of both minimal length and maximal momentum, there is no divergence in energy spectrum of a test particle. Moreover, the uncertainty principle is modified as a generalized uncertainty principle (GUP) [49, 50] to work on the effect of the quantum gravity which is applied to different areas. The important contribution of the GUP is to remove the divergences in physics. On the 
other hand, GUP can be used to modify Proca equation and Klein-Gordon equation to obtain the effects of the GUP on the Hawking temperature and check if it leaves remnants [51-54]. Nozari and Mehdipour [55] have discussed $\mathrm{BH}$ remnants and their cosmological constraints. Moreover, GUP is used to modify the thermodynamics of $\mathrm{N}$-dimensional Schwarzschild-Tangherlini black hole, speed of graviton, and the Entropic Force. Feng et al. [56] have studied the difference between the propagation speed of gravitons and the speed of light by using GUP. They have also investigated the modified speed of graviton by considering GUP. Rama [57] has studied the consequences of GUP which leads to varying speed of light and modified dispersion relations, which are likely to have implications for cosmology and black hole physics.

Since Maxwell, it was the dream of theoretical physicist to unify the fundamental forces in the nature in a single equation. Glashow, Weinberg, and Salam unified the theory of weak and electromagnetic interactions as an electroweak interaction in the 1960s. They assumed that the symmetry between the two different interactions would be clear at very large momentum transfers. However, at low energy, there is a mass difference between the photon and the $W_{+}, W_{-}$, and $Z_{0}$ bosons which break the symmetry.

This paper is organized as follows: In Section 2, we investigate the Hawking temperature of the black hole solutions surrounded by perfect fluid in Rastall theory using the tunneling of the massive vector particles. For this purpose we solve the equation of the motion of the Glashow-WeinbergSalam model using the semiclassical WKB approximation with Hamilton-Jacobi method. In Section 3. we use the GUPcorrected Proca equation to investigate the tunneling of massive uncharged vector particles for finding the corrected Hawking temperature of the black hole solutions surrounded by perfect fluid in Rastall theory. In Section 4, we conclude the paper with our results.

\section{Tunneling of Charged Massive Vector Bosons}

In this section, we study the tunneling of the charged massive bosons from the different types of black holes surrounded by the perfect fluids in Rastall theory.

2.1. The Black Hole Surrounded by the Dust Field in Rastall Theory. First we study the line element of the black hole surrounded by the dust field [58]:

$$
\begin{aligned}
d s^{2}= & -\left(1-\frac{2 M}{r}+\frac{Q^{2}}{r^{2}}-\frac{N_{d}}{r^{(1-6 \kappa \lambda) /(1-3 \kappa \lambda)}}\right) d t^{2} \\
& +\frac{d r^{2}}{1-2 M / r+Q^{2} / r^{2}-N_{d} / r^{(1-6 \kappa \lambda) /(1-3 \kappa \lambda)}} \\
& +r^{2}\left(d \theta^{2}+\sin ^{2} \theta d \phi^{2}\right)
\end{aligned}
$$

where $M$ is a mass of black hole, $\kappa$ and $\lambda$ are the Rastall geometric parameters, $N_{d}$ is the dust field structure parameter, and $Q$ is a charge of black hole. Now, we can rewrite (1) in the following form:

$$
d s^{2}=-G(r) d t^{2}+B(r) d r^{2}+C(r) d \theta^{2}+D(r) d \phi^{2},
$$

where $G(r), B(r), C(r)$, and $D(r)$ are given below:

$$
\begin{aligned}
& G(r)=1-\frac{2 M}{r}+\frac{Q^{2}}{r^{2}}-\frac{N_{d}}{r^{(1-6 \kappa \lambda) /(1-3 \kappa \lambda)},} \\
& C(r)=r^{2}, \\
& B(r)=\frac{1}{1-2 M / r+Q^{2} / r^{2}-N_{d} / r^{(1-6 \kappa \lambda) /(1-3 \kappa \lambda)},} \\
& D(r)=r^{2} \sin ^{2} \theta .
\end{aligned}
$$

The equation of motion for the Glashow-Weinberg-Salam model [59-61] is

$$
\begin{aligned}
& \frac{1}{\sqrt{-\mathbf{g}}} \partial_{\mu}\left(\sqrt{-\mathbf{g}} \Phi^{\nu \mu}\right)+\frac{m^{2}}{h^{2}} \Phi^{\nu}+\frac{i}{h} e A_{\mu} \Phi^{\nu \mu}+\frac{i}{h} e F^{\nu \mu} \Phi_{\mu} \\
& \quad=0
\end{aligned}
$$

where $|\mathbf{g}|$ is a coefficients matrix, $m$ is particles mass, and $\Phi^{\mu \nu}$ is antisymmetric tensor, since

$$
\begin{aligned}
\Phi_{\nu \mu} & =\partial_{\nu} \Phi_{\mu}-\partial_{\mu} \Phi_{\nu}+\frac{i}{h} e A_{\nu} \Phi_{\mu}-\frac{i}{h} e A_{\mu} \Phi_{\nu}, \\
F^{\mu \nu} & =\Delta^{\mu} A^{\nu}-\Delta^{\nu} A^{\mu},
\end{aligned}
$$

where $A_{\mu}$ is the vector potential of the charged black hole and $A_{0}$ and $A_{3}$ are the components of $A_{\mu}, e$ is the charge of the particle, and $\triangle_{\mu}$ is covariant derivative. The values of $\Phi^{\mu}$ and $\Phi^{\nu \mu}$ are given by

$$
\begin{aligned}
\Phi^{0} & =\frac{\Phi_{0}}{G}, \\
\Phi^{1} & =\frac{\Phi_{1}}{B}, \\
\Phi^{2} & =\frac{\Phi_{2}}{C}, \\
\Phi^{3} & =\frac{\Phi_{3}}{D}, \\
\Phi^{01} & =\frac{\Phi_{01}}{G B}, \\
\Phi^{02} & =\frac{\Phi_{02}}{G C}, \\
\Phi^{03} & =\frac{\Phi_{03}}{G D}, \\
\Phi^{12} & =\frac{\Phi_{12}}{B C}, \\
\Phi^{13} & =\frac{\Phi_{13}}{B D}, \\
\Phi^{23} & =\frac{\Phi_{23}}{C D} .
\end{aligned}
$$


Using WKB approximation for the wave function ansatz [62], i.e.,

$$
\Phi_{\nu}=c_{\nu} \exp \left[\frac{i}{\hbar} I_{0}(t, r, \theta, \phi)+\sum_{\zeta=1}^{\zeta=n} \hbar^{\zeta} I_{\zeta}(t, r, \theta, \phi)\right],
$$

to the Lagrangian (4) (where $I_{0}$ and $I_{i}$ correspond to particles action, for $i=1,2,3, \ldots)$ and neglecting the higher order terms, we get the following set of equations given below:

$$
\begin{aligned}
& \frac{1}{B}\left[c_{1}\left(\partial_{0} I_{0}\right)\left(\partial_{1} I_{0}\right)+e A_{0} c_{1}\left(\partial_{1} I_{0}\right)-c_{0}\left(\partial_{1} I_{0}\right)^{2}\right] \\
& +\frac{1}{C}\left[c_{2}\left(\partial_{0} I_{0}\right)\left(\partial_{2} I_{0}\right)-c_{0}\left(\partial_{2} I_{0}\right)^{2}+e A_{0} c_{2}\left(\partial_{2} I_{0}\right)\right] \\
& +\frac{1}{D}\left[c_{3}\left(\partial_{0} I_{0}\right)\left(\partial_{3} I_{0}\right)+e A_{0} c_{3}\left(\partial_{3} I_{0}\right)-c_{0}\left(\partial_{3} I_{0}\right)^{2}\right] \\
& +\frac{e A_{3}}{D}\left[c_{3}\left(\partial_{0} I_{0}\right)+e A_{0} c_{3}-c_{0}\left(\partial_{3} I_{0}\right)\right]-m^{2} c_{0}=0, \\
& \frac{1}{G(r)}\left[c_{0}\left(\partial_{0} I_{0}\right)\left(\partial_{1} I_{0}\right)-e A_{0} c_{1}\left(\partial_{0} I_{0}\right)-c_{1}\left(\partial_{0} I_{0}\right)^{2}\right] \\
& -\frac{1}{C}\left[c_{2}\left(\partial_{1} I_{0}\right)\left(\partial_{2} I_{0}\right)-c_{1}\left(\partial_{2} I_{0}\right)^{2}\right] \\
& +\frac{1}{D}\left[c_{3}\left(\partial_{1} I_{0}\right)\left(\partial_{3} I_{0}\right)-c_{1}\left(\partial_{3} I_{0}\right)-e A_{3} c_{1}\left(\partial_{3} I_{0}\right)\right] \\
& +\frac{e A_{3}}{D}\left[c_{3}\left(\left(\partial_{1} I_{0}\right)-e A_{3}\right) c_{1}-c_{1}\left(\partial_{3} I_{0}\right)\right]-m^{2} c_{1} \\
& =0 \text {, } \\
& \frac{1}{G(r)}\left[c_{0}\left(\partial_{0} I_{0}\right)\left(\partial_{2} I_{0}\right)-c_{2}\left(\partial_{0} I_{0}\right)^{2}-e A_{0}\left(\partial_{0} I_{0}\right) c_{2}\right] \\
& -\frac{1}{B}\left[c_{2}\left(\partial_{1} I_{0}\right)^{2}-c_{1}\left(\partial_{1} I_{0}\right)\left(\partial_{2} I_{0}\right)\right] \\
& -\frac{1}{D}\left[c_{3}\left(\partial_{2} I_{0}\right)\left(\partial_{3} I_{0}\right)-c_{2}\left(\partial_{3} I_{0}\right)^{2}-e A_{3} \mathcal{c}_{2}\left(\partial_{3} I_{0}\right)\right] \\
& +\frac{e A_{0}}{G(r)}\left[c_{0}\left(\partial_{2} I_{0}\right)-c_{2}\left(\partial_{0} I_{0}\right)-c_{2} e A_{0}\right] \\
& +\frac{e A_{3}}{D}\left[c_{3}\left(\partial_{2} I_{0}\right)-c_{2}\left(\partial_{3} I_{0}\right)-e A_{3} c_{2}\right]-m^{2} c_{2}=0, \\
& \frac{1}{G(r)}\left[c_{0}\left(\partial_{0} I_{0}\right)\left(\partial_{3} I_{0}\right)-c_{3}\left(\partial_{0} I_{0}\right)^{2}+e A_{3} c_{0}\left(\partial_{0} I_{0}\right)\right. \\
& \left.-e A_{0} c_{3}\left(\partial_{0} I_{0}\right)\right]+\frac{1}{B}\left[c_{1}\left(\partial_{1} I_{0}\right)\left(\partial_{3} I_{0}\right)-c_{3}\left(\partial_{1} I_{0}\right)^{2}\right. \\
& \left.+e A_{3} c_{1}\left(\partial_{1} I_{0}\right)\right]+\frac{1}{C}\left[c_{2}\left(\partial_{2} I_{0}\right)\left(\partial_{3} I_{0}\right)-\mathcal{c}_{3}\left(\partial_{2} I_{0}\right)^{2}\right. \\
& \left.+e A_{3} c_{2}\left(\partial_{2} I_{0}\right)\right]+\frac{e A_{0}}{G(r)}\left[c_{0}\left(\partial_{3} I_{0}\right)-c_{3}\left(\partial_{0} I_{0}\right)\right. \\
& \left.+e A_{3} c_{0}-e A_{0} c_{3}\right]-m^{2} c_{3}=0 .
\end{aligned}
$$

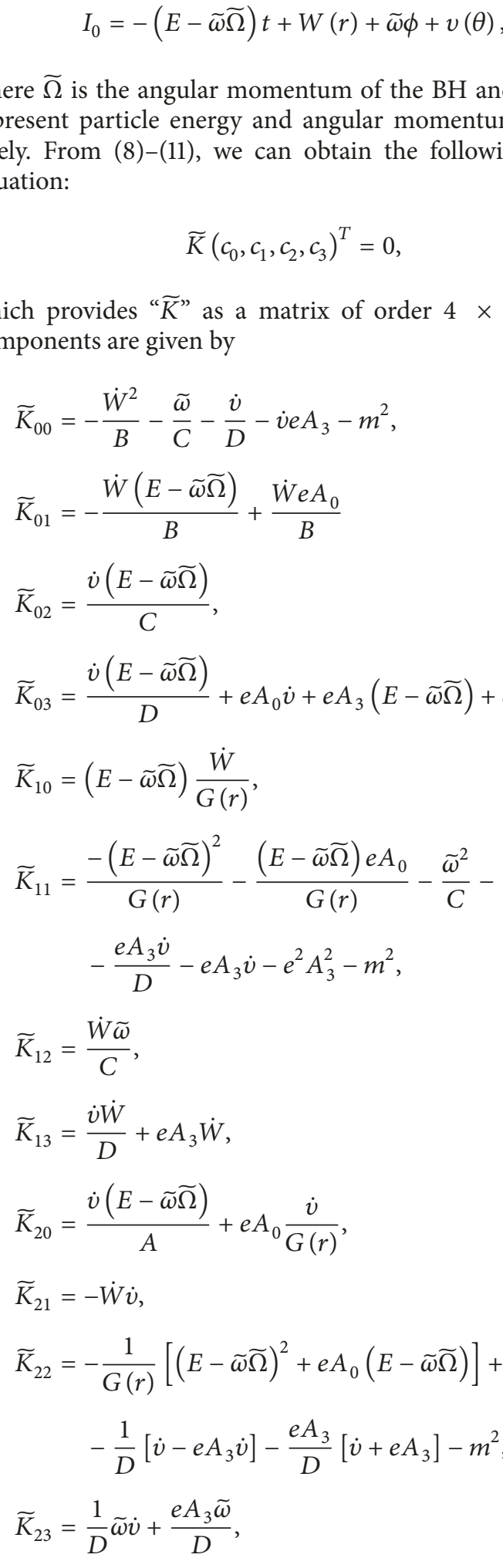

$$
\begin{aligned}
& \widetilde{K}_{30}=\frac{1}{G(r)}\left[(E-\widetilde{\omega} \widetilde{\Omega}) \dot{v}+e A_{3}(E-\widetilde{\omega} \widetilde{\Omega})\right] \\
& +\frac{e A_{0}}{G(r)}\left[\dot{v}+e A_{3}\right] \\
& \widetilde{K}_{00}=-\frac{\dot{W}^{2}}{B}-\frac{\widetilde{\omega}}{C}-\frac{\dot{v}}{D}-\dot{v} e A_{3}-m^{2}, \\
& \begin{array}{l}
\widetilde{K}_{01}=-\frac{\dot{W}(E-\widetilde{\omega} \widetilde{\Omega})}{B}+\frac{\dot{W} e A_{0}}{B} \\
\widetilde{K}_{02}=\frac{\dot{v}(E-\widetilde{\omega} \widetilde{\Omega})}{C}, \\
\widetilde{K}_{03}=\frac{\dot{v}(E-\widetilde{\omega} \widetilde{\Omega})}{D}+e A_{0} \dot{v}+e A_{3}(E-\widetilde{\omega} \widetilde{\Omega})+e A_{0},
\end{array} \\
& \widetilde{K}_{10}=(E-\widetilde{\omega} \widetilde{\Omega}) \frac{\dot{W}}{G(r)}, \\
& \widetilde{K}_{11}=\frac{-(E-\widetilde{\omega} \widetilde{\Omega})^{2}}{G(r)}-\frac{(E-\widetilde{\omega} \widetilde{\Omega}) e A_{0}}{G(r)}-\frac{\widetilde{\omega}^{2}}{C}-\frac{\dot{v}}{D} \\
& \widetilde{K}_{12}=\frac{\dot{W} \widetilde{\omega}}{C}, \\
& \widetilde{K}_{13}=\frac{\dot{v} \dot{W}}{D}+e A_{3} \dot{W}, \\
& \widetilde{K}_{20}=\frac{\dot{v}(E-\widetilde{\omega} \widetilde{\Omega})}{A}+e A_{0} \frac{\dot{v}}{G(r)}, \\
& \widetilde{K}_{21}=-\dot{W} \dot{v} \\
& \widetilde{K}_{22}=-\frac{1}{G(r)}\left[(E-\widetilde{\omega} \widetilde{\Omega})^{2}+e A_{0}(E-\widetilde{\omega} \widetilde{\Omega})\right]+\frac{1}{B} \dot{W}
\end{aligned}
$$

We can choose $I_{0}$ by using separation of variables technique, i.e.,

$$
I_{0}=-(E-\widetilde{\omega} \widetilde{\Omega}) t+W(r)+\widetilde{\omega} \phi+v(\theta),
$$

where $\widetilde{\Omega}$ is the angular momentum of the $\mathrm{BH}$ and $E$ and $\widetilde{\omega}$ represent particle energy and angular momentum, respecwe can obtain the following matrix equation:

which provides " $\widetilde{K}$ " as a matrix of order $4 \times 4$ and its components are given by 


$$
\begin{aligned}
\widetilde{K}_{31}= & \frac{1}{B}\left[\dot{W} \dot{v}+e A_{3} \dot{W}\right] \\
\widetilde{K}_{32}= & \frac{1}{C}\left[\widetilde{\omega} \dot{v}+e A_{3} \dot{v}\right] \\
\widetilde{K}_{33}= & -\frac{1}{G(r)}\left[(E-\widetilde{\omega} \widetilde{\Omega})^{2}+(E-\widetilde{\omega} \widetilde{\Omega}) e A_{0}\right]-\frac{1}{B} \dot{W}^{2} \\
& -\frac{\dot{v}^{2}}{C}-\frac{e A_{0}}{G(r)}\left[(E-\widetilde{\omega} \widetilde{\Omega})+e A_{0}\right]-m^{2},
\end{aligned}
$$

where $\dot{W}=\partial_{r} I_{0}, \dot{v}=\partial_{\theta} I_{0}$, and $\widetilde{\omega}=\partial_{\phi} I_{0}$. For the nontrivial solution $|\widetilde{\mathbf{K}}|=0$ and solving above equations one can yield

$$
\operatorname{Im} W^{ \pm}= \pm \int \sqrt{\frac{\left(E-e A_{0}-\widetilde{\omega} \widetilde{\Omega}-e A_{3}\right)^{2}+X}{G(r) B^{-1}}} d r
$$

where + and - represent the outgoing and incoming particles, respectively, whereas, " $X$ " is the function which can be defined as

$$
\begin{aligned}
X= & -\frac{G(r)}{C} \dot{v}^{2}-m^{2} G(r)+2 e A_{3}(E-\tilde{\omega} \widetilde{\Omega}) \\
& +2 e^{2} A_{0} A_{3}-e^{2} A_{3}^{2}
\end{aligned}
$$

and $\widetilde{\omega}$ is the angular velocity at event horizon.

By integrating (15) around the pole, we get

$$
\operatorname{Im} W^{ \pm}= \pm i \pi \frac{\left(E-e A_{0}-\widetilde{\omega} \widetilde{\Omega}-e A_{3}\right)}{2 \rho\left(r_{+}\right)}
$$

where the surface gravity $\kappa\left(r_{+}\right)$of the charged black hole is given by

$$
\kappa\left(r_{+}\right)=\left[\frac{2 M}{r^{2}}-\frac{2 Q^{2}}{r^{3}}+\frac{1-6 \kappa \lambda}{1-3 \kappa \lambda} N_{d} r^{3 \kappa \lambda /(3 \kappa \lambda-1)}\right]_{r=r_{+}}^{2} .
$$

The tunneling probability $\Gamma$ for outgoing charged vector particles can be obtained by

$$
\begin{aligned}
\Gamma\left(\operatorname{Im} W^{+}\right) & =\frac{\operatorname{Prob}[\text { emission }]}{\operatorname{Prob}[\text { absorption] }}=\frac{\exp \left[-2\left(\operatorname{Im} W^{+}+\operatorname{Im} \Phi\right)\right]}{\exp \left[-2\left(\operatorname{Im} W^{-}-\operatorname{Im} \Phi\right)\right]}=\exp \left[-4 \operatorname{Im} W^{+}\right] \\
& =\exp \left[-\frac{2 \pi\left(E-e A_{0}-\widetilde{\omega} \widetilde{\Omega}-e A_{3}\right)}{\left(2 M / r^{2}-2 Q^{2} / r^{3}+((1-6 \kappa \lambda) /(1-3 \kappa \lambda)) N_{d} r^{3 \kappa \lambda /(3 \kappa \lambda-1)}\right)^{2}}\right] .
\end{aligned}
$$

Now, we can calculate the $\widetilde{T}_{H}\left(\operatorname{Im} W^{+}\right)$by comparing the $\widetilde{\Gamma}\left(\operatorname{Im} W^{+}\right)$with the Boltzmann formula $\widetilde{\Gamma}_{B}\left(\operatorname{Im} W^{+}\right) \approx$ $e^{-\left(E-e A_{0}-\widetilde{\omega} \widetilde{\Omega}-e A_{3}\right) / \widetilde{T}_{H}\left(\operatorname{Im} W^{+}\right)}$, and we get

$$
\begin{aligned}
T_{H} & \left(\operatorname{Im} W^{+}\right) \\
& =\left[\frac{M}{\pi r^{2}}-\frac{Q^{2}}{\pi r^{3}}+\frac{1-6 \kappa \lambda}{2 \pi(1-3 \kappa \lambda)} N_{d} r^{3 \kappa \lambda /(3 \kappa \lambda-1)}\right]_{r=r_{+}}^{2} .
\end{aligned}
$$

The result shows that the $\widetilde{\Gamma}\left(\operatorname{Im} W^{+}\right)$is dependent on $r$, the vector potential components $\left(A_{0}\right.$ and $\left.A_{3}\right)$, energy $E$, angular momentum $\widetilde{\Omega}$, and mass of black hole $M ; \kappa$ and $\lambda$ are the Rastall geometric parameters; and $N_{d}$ and $Q$ are dust field structure parameter and charge of black hole, respectively.

2.2. The Black Hole Surrounded by the Radiation Field. Second example of the line element of black hole surrounded by the radiation field [58] is given below:

$$
\begin{aligned}
d s^{2}= & -\left(1-\frac{2 M}{r}+\frac{Q^{2}-N_{r}}{r^{2}}\right) d t^{2} \\
& +\frac{d r^{2}}{\left(1-2 M / r+\left(Q^{2}-N_{r}\right) / r^{2}\right)} \\
& +r^{2}\left(d \theta^{2}+\sin ^{2} \theta d \phi^{2}\right),
\end{aligned}
$$

where $M$ is a mass of black hole, $N_{r}$ is the negative radiation structure parameter, and $Q$ is a charge of black hole.

Following the procedure given in Section 2.1 for this line element, we can obtain the surface gravity $\kappa\left(r_{+}\right)$of this charged black hole surrounded by the radiation field in the following form:

$$
\kappa\left(r_{+}\right)=\left[\frac{2 M}{r^{2}}-\frac{2 Q^{2}}{r^{3}}-\frac{r^{2} \dot{N}_{r}-2 r N_{r}}{r^{4}}\right]_{r=r_{+}}^{2},
$$

where $\dot{N}_{r}=\partial N_{r} / \partial r$. The tunneling rate of particles can be calculated as

$$
\widetilde{\Gamma}\left(\operatorname{Im} W^{+}\right)=\exp \left[-\frac{4 \pi\left(E-e A_{\mu}-\widetilde{\omega} \widetilde{\Omega}\right) r^{6}}{\left[2 M r-2 Q^{2}-r \dot{N}_{r}+2 N_{r}\right]^{2}}\right]
$$

and the corresponding Hawking temperature at horizon can be obtained as

$$
\widetilde{T}\left(r_{+}\right)=\left.\frac{1}{4 \pi r^{6}}\left[2 M r-2 Q^{2}-r \dot{N}_{r}+2 N_{r}\right]^{2}\right|_{r=r_{+}} .
$$

This temperature depends on radiation structure parameter $N_{r}$, mass $M$, and black hole charge $Q$. 
2.3. The Black Hole Surrounded by the Quintessence Field. Third example of the line element of the black hole surrounded by the quintessence field [58] is given below:

$$
\begin{aligned}
d s^{2}= & -\left(1-\frac{2 M}{r}+\frac{Q^{2}}{r^{2}}-\frac{N_{q}}{r^{(-1-2 \kappa \lambda) /(1-\kappa \lambda)}}\right) d t^{2} \\
& +\frac{d r^{2}}{\left(1-2 M / r+Q^{2} / r^{2}-N_{q} / r^{(-1-2 \kappa \lambda) /(1-\kappa \lambda)}\right)} \\
& +r^{2}\left(d \theta^{2}+\sin ^{2} \theta d \phi^{2}\right),
\end{aligned}
$$

$$
\bar{\Gamma}=\exp \left[\frac{-4 \pi\left(E-e A_{\mu}-\widetilde{\omega} \widetilde{\Omega}\right)}{\left[2 M / r^{2}-2 Q^{2} / r^{3}+((1-6 \kappa \lambda) /(1-3 \kappa \lambda)) N_{d} r^{3 \kappa \lambda /(3 \kappa \lambda-1)}\right]^{2}}\right]
$$

and Hawking temperature

$$
\bar{T}=\left[\frac{\left(2 M / r^{2}-2 Q^{2} / r^{3}-((1+2 \kappa \lambda) /(1-\kappa \lambda)) N_{q} r^{3 \kappa \lambda /(1-\kappa \lambda-)}\right)^{2}}{4 \pi}\right]_{r=r_{+}}
$$

are derived and given in the above expressions. The Hawking temperature depends on $M, Q$, and $N_{q}$, i.e., quintessence field structure parameter, mass, and charge of black hole, respectively.

2.4. The Black Hole Surrounded by the Cosmological Constant Field. Fourth example of the line element of black hole surrounded by the cosmological constant field is given below [58]:

$$
\begin{aligned}
d s^{2}= & -\left(1-\frac{2 M}{r}+\frac{Q^{2}}{r^{2}}-N_{c} r^{2}\right) d t^{2} \\
& +\frac{d r^{2}}{\left(1-2 M / r+Q^{2} / r^{2}-N_{c} r^{2}\right)} \\
& +r^{2}\left(d \theta^{2}+\sin ^{2} \theta d \phi^{2}\right),
\end{aligned}
$$

where $N_{c}$ is a cosmological constant field structure parameter. For this black hole, the surface gravity at outer horizon is obtained by following the above-mentioned similar procedure; i.e.,

$$
\kappa\left(r_{+}\right)=\left[\frac{2 M}{r^{2}}-\frac{2 Q^{2}}{r^{3}}-2 N_{c} r\right]_{r=r_{+}}^{2} .
$$

Moreover, the required tunneling probability of particles

$$
\widehat{\Gamma}=\exp \left[\frac{-4 \pi\left(E-e A_{\mu}-\widetilde{\omega} \widetilde{\Omega}\right)}{\left(2 M / r^{2}-2 Q^{2} / r^{3}-2 N_{c} r\right)^{2}}\right],
$$

and their corresponding Hawking temperature is calculated in the following expression:

$$
\widehat{T}=\left[\frac{\left(2 M / r^{2}-2 Q^{2} / r^{3}-2 N_{c} r\right)^{2}}{4 \pi}\right]_{r=r_{+}} .
$$

This temperature depends on $N_{c}, M$, and $Q$, i.e., cosmological constant field structure parameter, mass, and charge of black hole, respectively.

2.5. The Black Hole Surrounded by the Phantom Field. Last example of the line element of black hole surrounded by the phantom field is [58]

$$
\begin{aligned}
d s^{2}= & -\left(1-\frac{2 M}{r}+\frac{Q^{2}}{r^{2}}-\frac{N_{p}}{r^{(-3+2 \kappa \lambda) /(1+\kappa \lambda)}}\right) d t^{2} \\
& +\frac{d r^{2}}{\left(1-2 M / r+Q^{2} / r^{2}-N_{p} / r^{(-3+2 \kappa \lambda) /(1+\kappa \lambda)}\right)} \\
& +r^{2}\left(d \theta^{2}+\sin ^{2} \theta d \phi^{2}\right)
\end{aligned}
$$

where $N_{p}$ is a phantom field structure parameter. For vector potential $A_{\mu}$ of this black hole, the surface gravity can be derived as

$$
\begin{aligned}
& \kappa\left(r_{+}\right) \\
& =\left[\frac{2 M}{r^{2}}-\frac{2 Q^{2}}{r^{3}}-\left(\frac{3-2 \kappa \lambda}{1+\kappa \lambda}\right) N_{p} r^{(2-3 \kappa \lambda) /(1+\kappa \lambda)}\right]^{2} .
\end{aligned}
$$


The tunneling probability of particles

$$
\check{\Gamma}=\exp \left[\frac{-4 \pi\left(E-e A_{\mu}-\widetilde{\omega} \widetilde{\Omega}\right)}{\left(2 M / r^{2}-2 Q^{2} / r^{3}-((3-2 \kappa \lambda) /(1+\kappa \lambda)) N_{p} r^{(2-3 \kappa \lambda) /(1+\kappa \lambda)}\right)^{2}}\right]
$$

and the required Hawking temperature of particles can be obtained as given below:

$$
\check{T}=\left[\frac{\left(2 M / r^{2}-2 Q^{2} / r^{3}-((3-2 \kappa \lambda) /(1+\kappa \lambda)) N_{p} r^{(2-3 \kappa \lambda) /(1+\kappa \lambda)}\right)^{2}}{4 \pi}\right]_{r=r_{+}} .
$$

The Hawking temperature depends on $M, Q$, and $N_{p}$; these are phantom field structure parameter, mass, and charge of black hole, respectively.

\section{GUP-Corrected Proca Equation and the Corrected Hawking Temperature}

In this section, we focus on the effect of the GUP on the tunneling of massive uncharged vector particles from the black hole solutions surrounded by perfect fluid in Rastall theory. Firstly we use the GUP-corrected Lagrangian for the massive uncharged vector field $\psi_{\mu}$ given by [63]

$$
\begin{aligned}
L_{G U P}= & -\frac{1}{2}\left(D_{\mu} \psi_{\nu}-D_{\nu} \psi_{\mu}\right)\left(D^{\mu} \psi^{\nu}-D^{\nu} \psi^{\mu}\right) \\
& -\frac{m_{W}^{2}}{\hbar^{2}} \psi_{\mu} \psi^{\mu} .
\end{aligned}
$$

One can derive the equation of the motion for the GUPcorrected Lagrangian of massive uncharged vector field as follows [63]:

$$
\begin{aligned}
\partial_{\mu}\left(\sqrt{-g} \psi^{\mu \nu}\right)-\sqrt{-g} \frac{m_{W}^{2}}{\hbar^{2}} \psi^{\nu} \\
+\beta \hbar^{2} \partial_{0} \partial_{0} \partial_{0}\left(\sqrt{-g} g^{00} \psi^{0 v}\right) \\
-\beta \hbar^{2} \partial_{i} \partial_{i} \partial_{i}\left(\sqrt{-g} g^{i i} \psi^{i v}\right)=0,
\end{aligned}
$$

with

$$
\psi_{\mu \nu}=\left(1-\beta \hbar^{2} \partial_{\mu}^{2}\right) \partial_{\mu} \psi_{\nu}-\left(1-\beta \hbar^{2} \partial_{\nu}^{2}\right) \partial_{\nu} \psi_{\mu}
$$

It is noted that we use the Latin indices for the modified tensor $\psi_{i \mu}$ as follows: $i=1,2,3$; on the other hand, for $\psi_{0 \mu}$, we use the 0 for the time coordinate. Moreover, we note that $\beta=$ $1 /\left(3 M_{f}^{2}\right)$, where $M_{f}$ is the Planck mass and $m_{W}$ stands for the mass of the particle.
3.1. The Black Hole Surrounded by the Dust Field in Rastall Theory. The metric is given by

$$
\begin{aligned}
d s^{2}= & -G(r)(r) d t^{2}+B(r) d r^{2}+C(r) d \theta^{2} \\
& +D(r) d \phi^{2},
\end{aligned}
$$

where $G(r), B(r), C(r)$, and $D(r)$ are given below:

$$
\begin{aligned}
G(r) & =1-\frac{2 M}{r}+\frac{Q^{2}}{r^{2}}-\frac{N_{d}}{r^{(1-6 \kappa \lambda) /(1-3 \kappa \lambda)},} \\
C(r) & =r^{2}, \\
B(r) & =\frac{1}{G(r)} \\
& =\frac{1}{1-2 M / r+Q^{2} / r^{2}-N_{d} / r^{(1-6 \kappa \lambda) /(1-3 \kappa \lambda)},} \\
D(r) & =r^{2} \sin ^{2} \theta .
\end{aligned}
$$

Using the WKB method, we define the $\psi_{\mu}$ as follows:

$$
\Psi_{\mu}=c_{\mu}(t, r, \theta, \phi) \exp \left[\frac{i}{\hbar} I(t, r, \theta, \phi)\right],
$$

where $I$ is defined as

$$
\begin{aligned}
I(t, r, \theta, \phi)= & I_{0}(t, r, \theta, \phi)+\hbar I_{1}(t, r, \theta, \phi) \\
& +\hbar^{2} I_{2}(t, r, \theta, \phi)+\cdots .
\end{aligned}
$$

We use (43), (44), and the metric (40) into (38), and then we only consider the lowest order terms in $\hbar$ to calculate the equations with the corresponding coefficients $c_{\mu}$ :

$$
\begin{aligned}
& G(r)\left[c_{0}\left(\partial_{r} I_{0}\right)^{2} \mathscr{A}_{1}^{2}-c_{1}\left(\partial_{r} I_{0}\right)\left(\partial_{t} I_{0}\right) \mathscr{A}_{1} \mathscr{A}_{0}\right] \\
& +\frac{1}{C(r)}\left[c_{0}\left(\partial_{\theta} I_{0}\right)^{2} \mathscr{A}_{2}^{2}-c_{2}\left(\partial_{\theta} I_{0}\right)\left(\partial_{t} I_{0}\right) \mathscr{A}_{2} \mathscr{A}_{0}\right] \\
& \quad+\frac{1}{D(r)}\left[c_{0}\left(\partial_{\phi} I_{0}\right)^{2} \mathscr{A}_{3}^{2}-c_{3}\left(\partial_{\phi} I_{0}\right)\left(\partial_{t} I_{0}\right) \mathscr{A}_{3} \mathscr{A}_{0}\right] \\
& \quad+c_{0} m_{W}^{2}=0,
\end{aligned}
$$




$$
\begin{aligned}
- & \frac{1}{G(r)}\left[c_{1}\left(\partial_{t} I_{0}\right)^{2} \mathscr{A}_{0}^{2}-c_{0}\left(\partial_{t} I_{0}\right)\left(\partial_{r} I_{0}\right) \mathscr{A}_{0} \mathscr{A}_{1}\right] \\
& +\frac{1}{C(r)}\left[c 1\left(\partial_{\theta} I_{0}\right)^{2} \mathscr{A}_{2}^{2}-c_{2}\left(\partial_{\theta} I_{0}\right)\left(\partial_{r} I_{0}\right) \mathscr{A}_{2} \mathscr{A}_{1}\right] \\
& +\frac{1}{D(r)}\left[c_{1}\left(\partial_{\phi} I_{0}\right)^{2} \mathscr{A}_{3}^{2}-c_{3}\left(\partial_{\phi} I_{0}\right)\left(\partial_{r} I_{0}\right) A_{3} \mathscr{A}_{1}\right] \\
& +c_{1} m_{W}^{2}=0 \\
- & \frac{1}{G(r)}\left[c_{2}\left(\partial_{t} I_{0}\right)^{2} \mathscr{A}_{0}^{2}-c_{0}\left(\partial_{t} I_{0}\right)\left(\partial_{\theta} I_{0}\right) \mathscr{A}_{0} \mathscr{A}_{2}\right] \\
& +G(r)\left[c_{2}\left(\partial_{r} I_{0}\right)^{2} \mathscr{A}_{1}^{2}-c_{1}\left(\partial_{r} I_{0}\right)\left(\partial_{\theta} I_{0}\right) \mathscr{A}_{1} \mathscr{A}_{2}\right] \\
& +\frac{1}{D(r)}\left[c_{2}\left(\partial_{\phi} I_{0}\right)^{2} \mathscr{A}_{3}^{2}-c_{3}\left(\partial_{\phi} I_{0}\right)\left(\partial_{\theta} I_{0}\right) \mathscr{A}_{3} \mathscr{A}_{2}\right] \\
& +c_{2} m_{W}^{2}=0, \\
- & \frac{1}{G(r)}\left[c_{3}\left(\partial_{t} I_{0}\right)^{2} \mathscr{A}_{0}^{2}-c_{0}\left(\partial_{t} I_{0}\right)\left(\partial_{\phi} I_{0}\right) \mathscr{A}_{0} \mathscr{A}_{3}\right] \\
& +G(r)\left[c_{3}\left(\partial_{r} I_{0}\right)^{2} \mathscr{A}_{1}^{2}-c_{1}\left(\partial_{r} I_{0}\right)\left(\partial_{\phi} I_{0}\right) \mathscr{A}_{1} \mathscr{A}_{3}\right] \\
& +\frac{1}{C(r)}\left[c_{3}\left(\partial_{\theta} I_{0}\right)^{2} \mathscr{A}_{2}^{2}-c_{2}\left(\partial_{\theta} I_{0}\right)\left(\partial_{\phi} I_{0}\right) \mathscr{A}_{2} \mathscr{A}_{3}\right] \\
& +c_{3} m_{W}^{2}=0
\end{aligned}
$$

where the $\mathscr{A}_{\mu}$ s are defined as

$$
\begin{aligned}
& \mathscr{A}_{0}=1+\beta \frac{1}{G(r)}\left(\partial_{t} S_{0}\right)^{2}, \\
& \mathscr{A}_{1}=1+\beta G(r)\left(\partial_{r} S_{0}\right)^{2}, \\
& \mathscr{A}_{2}=1+\beta \frac{1}{C(r)}\left(\partial_{\theta} S_{0}\right)^{2}, \\
& \mathscr{A}_{3}=1+\beta \frac{1}{D(r)}\left(\partial_{\phi} S_{0}\right)^{2} .
\end{aligned}
$$

Using the semiclassical Hamilton-Jacobi method with WKB ansatz, we separate the variables as follows:

$$
I_{0}=-E t+R(r)+\Theta(\theta, \phi)+k .
$$

Note that the energy of the radiated particle is defined with $E$. Afterwards, we obtain a matrix equation as follows:

$$
\cup\left(c_{0}, c_{1}, c_{2}, c_{3}\right)^{T}=0,
$$

where $U$ is a $4 \times 4$ matrix, the elements of which are

$$
\begin{aligned}
\cup_{11} & =G(r) R^{\prime 2} \mathscr{A}_{1}^{2}+\frac{J_{\theta}^{2}}{C(r)} \mathscr{A}_{2}^{2}+\frac{J_{\phi}^{2}}{D(r)} \mathscr{A}_{3}^{2}+m_{W}^{2}, \\
\cup_{12} & =-G(r) R^{\prime}(-E) \mathscr{A}_{1} \mathscr{A}_{0},
\end{aligned}
$$

$$
\begin{aligned}
& \mathrm{U}_{13}=-\frac{J_{\theta}(-E)}{C(r)} \mathscr{A}_{2} \mathscr{A}_{0}, \\
& \cup_{14}=-\frac{J_{\phi}(-E)}{D(r)} \mathscr{A}_{3} \mathscr{A}_{0}, \\
& \mathrm{U}_{21}=\frac{(-E) R^{\prime}}{G(r)} \mathscr{A}_{0} \mathscr{A}_{1} \text {, } \\
& \cup_{22}=-\frac{(-E)^{2}}{G(r)} \mathscr{A}_{0}^{2}+\frac{J_{\theta}^{2}}{C(r)} \mathscr{A}_{2}^{2}+\frac{J_{\phi}^{2}}{D(r)} \mathscr{A}_{3}^{2}+m_{W}^{2}, \\
& \cup_{23}=-\frac{J_{\theta} R^{\prime}}{C(r)} \mathscr{A}_{2} \mathscr{A}_{1} \text {, } \\
& \cup_{24}=-\frac{J_{\phi} R^{\prime}}{D(r)} \mathscr{A}_{3} \mathscr{A}_{1} \text {, } \\
& \cup_{31}=\frac{-E J_{\theta}}{G(r)} \mathscr{A}_{0} \mathscr{A}_{2} \text {, } \\
& \cup_{32}=-G(r) R^{\prime} J_{\theta} \mathscr{A}_{1} \mathscr{A}_{2} \text {, } \\
& \cup_{33}=-\frac{(-E)^{2}}{G(r)} \mathscr{A}_{0}^{2}+G(r) R^{\prime 2} \mathscr{A}_{1}^{2}+\frac{J_{\phi}^{2}}{D(r)} \mathscr{A}_{3}^{2}+m_{W}^{2} \text {, } \\
& \cup_{34}=-\frac{J_{\theta} J_{\phi}}{D(r)} \mathscr{A}_{3} \mathscr{A}_{2} \text {, } \\
& \mathrm{U}_{41}=\frac{(-E) J_{\phi}}{G(r)} \mathscr{A}_{0} \mathscr{A}_{3}, \\
& \cup_{42}=-G(r) R^{\prime} J_{\phi} \mathscr{A}_{1} \mathscr{A}_{3} \text {, } \\
& \mathrm{U}_{43}=-\frac{J_{\theta} J_{\phi}}{C(r)} \mathscr{A}_{2} \mathscr{A}_{3} \text {, } \\
& \cup_{44}=-\frac{(-E)^{2}}{G(r)} \mathscr{A}_{0}^{2}+G(r) R^{\prime 2} A_{1}^{2}+\frac{J_{\theta}^{2}}{C(r)} \mathscr{A}_{2}^{2}+m_{W}^{2},
\end{aligned}
$$

where $R^{\prime}=\partial_{r} R, J_{\theta}=\partial_{\theta} \Theta$, and $J_{\phi}=\partial_{\phi} \Theta$.

It is noted that, for the condition of $\operatorname{det} U=0$, we find the nontrivial solution of (51). First, we consider only the lowest order terms of $\beta$ and then calculate the $\operatorname{det} U=0$. Our main aim is to obtain the radial part of the equation so that we integrate it using the complex integral method around the event horizon as follows:

$$
\begin{aligned}
& \operatorname{Im} R_{ \pm}(r) \\
& \quad= \pm \operatorname{Im} \int d r \sqrt{-\frac{m^{2}}{G(r)}+\frac{E^{2}}{G(r)^{2}}-\frac{J_{\theta}^{2}+J_{\phi}^{2}}{G(r) D(r)}}(1 \\
& \left.\quad+\frac{\mathscr{T}_{1}}{\mathscr{T}_{2}} \beta\right),
\end{aligned}
$$

where

$$
\begin{aligned}
\mathscr{T}_{1}= & -3 G(r) m^{4} C(r)+6 m^{2} C(r)(E)^{2} \\
& -6 G(r) m^{2}\left(J_{\theta}^{2}+\frac{J_{\phi}^{2}}{D(r)}\right)-\frac{6 G(r) J_{\theta}^{4}}{C(r)}
\end{aligned}
$$




$$
\begin{aligned}
+ & 6(E)^{2}\left(J_{\theta}^{2}+\frac{J_{\phi}^{2}}{D(r)}\right)-\frac{7 G(r) J_{\theta}^{2} J_{\phi}^{2}}{D(r)} \\
& -\frac{3 G(r) J_{\theta}^{4} J_{\phi}^{2}}{2 m^{2} D(r)^{2}}-\frac{5 G(r) J_{\phi}^{4} \csc ^{4} \theta}{C(r)} \\
& +\frac{3 G(r) J_{\theta}^{2} J_{\phi}^{4}}{2 m^{2} D(r)^{2}} \\
\mathscr{T}_{2}= & -G(r) m^{2} r^{2}+r^{2}(E)^{2}-G(r)\left(J_{\theta}^{2}+\frac{J_{\phi}^{2}}{D(r)}\right) .
\end{aligned}
$$

We rewrite the metric close to the event horizon to obtain solution for the integral:

$$
G\left(r_{h}\right) \approx \frac{\Delta_{, r}\left(r_{h}\right)}{r_{h}^{2}}\left(r-r_{h}\right)
$$

Afterwards we manage to obtain the solution of the integral (53) for the radial part as follows:

$$
\operatorname{Im} R_{ \pm}(r)= \pm i \pi \frac{r_{h}^{2}}{\Delta_{, r}\left(r_{h}\right)}(E) \times(1+\beta \Xi)
$$

where $\Xi=6 m^{2}+\left(6 / r_{h}^{2}\right)\left(J_{\theta}^{2}+J_{\phi}^{2} \csc ^{2} \theta\right)$.

It is quite clear that $\Xi>0$. We note that $R_{+}$represents the radial function for the outgoing particles and $R_{-}$is for the ingoing particles. Thus, the tunneling rate of $W$ bosons near the event horizon is

$$
\begin{aligned}
\Gamma & =\frac{P_{\text {outgoing }}}{P_{\text {ingoing }}}=\frac{\exp \left[-(2 / \hbar)\left(\operatorname{Im} R_{+}+\operatorname{Im} \Theta\right)\right]}{\exp \left[-(2 / \hbar)\left(\operatorname{Im} R_{-}+\operatorname{Im} \Theta\right)\right]} \\
& =\exp \left[-\frac{4}{\hbar} \operatorname{Im} R_{+}\right] \\
& =\exp \left[-\frac{4 \pi}{\hbar} \frac{r_{h}^{2}}{\Delta_{, r}\left(r_{h}\right)}(E) \times(1+\beta \Xi)\right] .
\end{aligned}
$$

If we set $\hbar=1$, we find the corrected Hawking temperature as follows:

$$
T_{e-H}=\frac{\Delta_{, r}\left(r_{h}\right)}{4 \pi r_{h}^{2}(1+\beta \Xi)}=T_{0}(1-\beta \Xi),
$$

where $T_{0}=\left[M / \pi r^{2}-Q^{2} / \pi r^{3}+((1-6 \kappa \lambda) / 2 \pi(1-3 \kappa \lambda))\right.$ $\left.N_{d} r^{3 \kappa \lambda /(3 \kappa \lambda-1)}\right]_{r=r_{+}}^{2}$ is the original Hawking temperature of a corresponding black hole. We find the corrected Hawking temperature with the effect of quantum gravity. In addition, the Hawking temperature is increased if one uses the quantum gravity effects, but then these effects are canceled in some point and black hole remnants occur.

3.2. The Black Hole Surrounded by the Radiation Field. Following the procedure given in Section 2.1 for this line element, we obtain the corrected Hawking temperature with the effect of quantum gravity for this charged black hole surrounded by the radiation field in the following form:

$$
T_{e-H}=\frac{\Delta_{, r}\left(r_{h}\right)}{4 \pi r_{h}^{2}(1+\beta \Xi)}=T_{0}(1-\beta \Xi),
$$

where original Hawking temperature is

$$
T_{0}=\left.\frac{1}{4 \pi r^{6}}\left[2 M r-2 Q^{2}-r \dot{N}_{r}+2 N_{r}\right]^{2}\right|_{r=r_{+}} .
$$

This temperature depends on radiation structure parameter $N_{r}$, mass $M$, and black hole charge $Q$. Moreover, the quantum effects explicitly counteract the temperature increases during evaporation, which will cancel it out at some point. Naturally, black hole remnants will be left.

3.3. The Black Hole Surrounded by the Quintessence Field. By following the same process, we calculate the corrected Hawking temperature under the effect of quantum gravity as follows:

$$
T_{e-H}=\frac{\Delta_{, r}\left(r_{h}\right)}{4 \pi r_{h}^{2}(1+\beta \Xi)}=T_{0}(1-\beta \Xi),
$$

with the original Hawking temperature

$$
T_{0}=\left[\frac{\left(2 M / r^{2}-2 Q^{2} / r^{3}-((1+2 \kappa \lambda) /(1-\kappa \lambda)) N_{q} r^{3 \kappa \lambda /(1-\kappa \lambda-)}\right)^{2}}{4 \pi}\right]_{r=r_{+}},
$$

derived and given in above expressions. The Hawking temperature depends on $M, Q$, and $N_{q}$, i.e., quintessence field structure parameter, mass, and charge of black hole, respectively. Naturally, black hole remnants will be left.

\subsection{The Black Hole Surrounded by the Cosmological Constant} Field. One can repeat the process just for this black hole to calculate the corresponding corrected Hawking temperature with the quantum gravity effects as follows:

$$
T_{e-H}=\frac{\Delta_{, r}\left(r_{h}\right)}{4 \pi r_{h}^{2}(1+\beta \Xi)}=T_{0}(1-\beta \Xi)
$$

with

$$
T_{0}=\left[\frac{\left(2 M / r^{2}-2 Q^{2} / r^{3}-2 N_{c} r\right)^{2}}{4 \pi}\right]_{r=r_{+}} .
$$


This temperature depends on $N_{c}, M$, and Q, i.e., cosmological constant field structure parameter, mass, and charge of black hole, respectively. Again here, remnants are left.

3.5. The Black Hole Surrounded by the Phantom Field. Last example is the line element of black hole surrounded by the phantom field. Now we again repeat the same process to obtain the following corrected Hawking temperature:

$$
T_{e-H}=\frac{\Delta_{, r}\left(r_{h}\right)}{4 \pi r_{h}^{2}(1+\beta \Xi)}=T_{0}(1-\beta \Xi),
$$

with

$$
\check{T}=\left[\frac{\left(2 M / r^{2}-2 Q^{2} / r^{3}-((3-2 \kappa \lambda) /(1+\kappa \lambda)) N_{p} r^{(2-3 \kappa \lambda) /(1+\kappa \lambda)}\right)^{2}}{4 \pi}\right]_{r=r_{+}} .
$$

The Hawking temperature depends on $M, Q$, and $N_{p}$; these are phantom field structure parameter, mass, and charge of black hole, respectively.

\section{Conclusions}

In this research paper, we have successfully analyzed the GUP-corrected Hawking temperature of $W^{ \pm}$boson vector particles using the equation of motion for the GlashowWeinberg-Salam model. First of all, we analyzed the modified Hamilton-Jacobi equation by resolving the modified Lagrangian equation utilized for the magnetized particles in the space-time. We have analyzed the GUP effect on the radiation of black holes surrounded by perfect fluid in Rastall theory.

As the original Hawing radiation, the Hawking temperature $T_{H}$ of the black holes is associated with its mass $M$ and charged $Q$. However, these results indicated that the effect of quantum gravity is counted and the behavior of the tunneling boson vector particle on the event horizon is observed from the original event. The Hawking temperature $T_{H}$ and tunneling probability $\Gamma$ quantities are not just sensitively dependent on the mass $M$ and charged $Q$ of the black hole. The Hawking temperature $T_{H}$, tunneling probability $\Gamma$, and surface gravity $\kappa$ are only dependent on the geometry (structure parameter) of black hole. Moreover, the corrected Hawking temperature $T_{e-H}=T_{0}(1-\beta \Xi)$ has been calculated with the effect of quantum gravity. The Hawking temperature is increased if one uses the quantum gravity effects, but then these effects are canceled in some point and black hole remnants occur.

\section{Data Availability}

No data were used to support this study.

\section{Conflicts of Interest}

The authors declare that there are no conflicts of interest regarding the publication of this paper.

\section{Acknowledgments}

This work is supported by Comisión Nacional de Ciencias y Tecnología of Chile (CONICYT) through FONDECYT Grant N 3170035 (Ali Övgün).

\section{References}

[1] D. Kubizňák and R. B. Mann, " $P-V$ criticality of charged AdS black holes," Journal of High Energy Physics, vol. 33, 2012.

[2] D. Kubizňák and R. B. Mann, "Black hole chemistry," Canadian Journal of Physics, vol. 93, no. 9, pp. 999-1002, 2015.

[3] D. Kubiznak, R. B. Mann, and M. Teo, "Black hole chemistry: thermodynamics with lambda," Classical and Quantum Gravity, vol. 34, no. 6, Article ID 063001, 2017.

[4] J. D. Bekenstein, "Generalized second law of thermodynamics in black-hole physics," Physical Review D: Particles, Fields, Gravitation and Cosmology, vol. 9, no. 12, pp. 3292-3300, 1974.

[5] S. W. Hawking, "Black hole explosions?" Nature, vol. 248, no. 5443, pp. 30-31, 1974.

[6] S. W. Hawking, "Particle creation by black holes," Communications in Mathematical Physics, vol. 43, no. 3, pp. 199-220, 1975.

[7] S. W. Hawking, "Erratum: 'Particle creation by black holes," Communications in Mathematical Physics, vol. 46, no. 2, p. 206, 1976.

[8] S. W. Hawking, M. J. Perry, and A. Strominger, "Soft Hair on Black Holes," Physical Review Letters, vol. 116, no. 23, Article ID 231301, 2016.

[9] M. K. Parikh and F. Wilczek, "Hawking radiation as tunneling," Physical Review Letters, vol. 85, no. 24, pp. 5042-5045, 2000.

[10] R. Kerner and R. B. Mann, "Tunnelling, temperature, and Taub-NUT black holes," Physical Review D: Particles, Fields, Gravitation and Cosmology, vol. 73, no. 10, Article ID 104010, 2006.

[11] R. Kerner and R. B. Mann, “Tunnelling from Gödel black holes," Physical Review D: Particles, Fields, Gravitation and Cosmology, vol. 75, no. 8, Article ID 084022, 2007.

[12] R. Banerjee and B. R. Majhi, "Quantum tunneling beyond semiclassical approximation," Journal of High Energy Physics, vol. 2008, article 095, 2008.

[13] E. T. Akhmedov, V. Akhmedova, and D. Singleton, "Hawking temperature in the tunneling picture," Physics Letters B, vol. 642, no. 1-2, pp. 124-128, 2006.

[14] E. T. Akhmedov, V. Akhmedova, D. Singleton, and T. Pilling, "Thermal radiation of various gravitational backgrounds," International Journal of Modern Physics A, vol. 22, no. 8-9, pp. 17051715, 2007.

[15] V. E. Akhmedova, T. Pilling, A. de Gill, and D. Singlton, "Tunneling/WKB and anomaly methods for Rindler and de sitter space-times," Theoretical and Mathematical Physics, vol. 163, no. 3, pp. 774-781, 2010. 
[16] V. Akhmedova, T. Pilling, A. de Gill, and D. Singleton, “Temporal contribution to gravitational WKB-like calculations," Physics Letters B, vol. 666, no. 3, pp. 269-271, 2008.

[17] K. Nozari and S. H. Mehdipour, "Hawking radiation as quantum tunneling from a noncommutative Schwarzschild black hole," Classical and Quantum Gravity, vol. 25, no. 17, Article ID 175015, 2008.

[18] K. Nozari and S. Saghafi, "Natural cutoffs and quantum tunneling from black hole horizon," Journal of High Energy Physics, vol. 2012, article 005, 2012.

[19] M. Angheben, M. Nadalini, L. Vanzo, and S. Zerbini, "Hawking radiation as tunneling for extremal and rotating black holes," Journal of High Energy Physics, vol. 2005, no. 5, article 014, 2005.

[20] S. I. Kruglov, "Black hole emission of vector particles in $(1+1)$ dimensions," International Journal of Modern Physics A, vol. 29, no. 22, Article ID 1450118, 2014.

[21] S. I. Kruglov, "Black hole radiation of spin-1 particles in $(1+2)$ dimensions," International Journal of Modern Physics A, vol. 29, no. 39, Article ID 1450203, 2014.

[22] X. M. Kuang, J. Saavedra, and A. Övgün, "The effect of the Gauss-Bonnet term on Hawking radiation from arbitrary dimensional black brane," The European Physical Journal C, vol. 77, article 613, 2017.

[23] I. Sakalli, A. Ovgun, and Lett. Europhys, "Hawking radiation and deflection of light from Rindler modified Schwarzschild black hole," EPL (Europhysics Letters), vol. 118, no. 6, Article ID 60006, 2017.

[24] K. Jusufi, İ. Sakallı, and A. Övgün, "Quantum tunneling and quasinormal modes in the spacetime of the Alcubierre warp drive," General Relativity and Gravitation, vol. 50, article 10, 2018.

[25] I. Sakalli, A. Övgün, and K. Jusuf, "GUP assisted Hawking radiation of rotating acoustic black holes," Astrophysics and Space Science. An International Journal of Astronomy, Astrophysics and Space Science, vol. 361, no. 10, article 330, 2016.

[26] A. Caliskan, S. O. Kara, and A. Ozansoy, "The BekensteinHawking corpuscular cascading from the back-reacted black hole," Advances in High Energy Physics, vol. 2017, Article ID 1573904, pp. 1-9, 2017.

[27] I. Sakalli and A. Övgün, "Black hole radiation of massive spin2 particles in (3+1) dimensions," The European Physical Journal Plus, vol. 131, no. 6, article 184, 2016.

[28] A. Övgün and K. Jusufi, "Massive vector particles tunneling from noncommutative charged black holes and its gupcorrected thermodynamics," The European Physical Journal Plus, vol. 131, no. 5, article 177, 2016.

[29] I. Sakalli and A. Ovgun, "Quantum tunneling of massive spin1 particles from non-stationary metrics," General Relativity and Gravitation, vol. 48, article 1, 2016.

[30] I. Sakalli and A. Ovgun, "Tunnelling of vector particles from Lorentzian wormholes in 3+1 dimensions," The European Physical Journal Plus, vol. 130, article 110, 2015.

[31] A. Övgün, I. Sakalli, and J. Saavedra, "Quasinormal modes of a schwarzschild black hole immersed in an electromagnetic universe," 2017, https://arxiv.org/abs/1708.08331.

[32] I. Sakalli, "Analytical solutions in rotating linear dilaton black holes: resonant frequencies, quantization, greybody factor, and Hawking radiation," Physical Review D: Particles, Fields, Gravitation and Cosmology, vol. 94, no. 8, Article ID 084040, 2016.

[33] T. I. Singh, I. A. Meitei, and K. Y. Singh, "Quantum gravity effects on hawking radiation of schwarzschild-de sitter black holes," International Journal of Theoretical Physics, vol. 56, no. 8, pp. 2640-2650, 2017.

[34] J. Pu and Y. Han, "On Hawking radiation via tunneling from the Reissner-Nordström-de Sitter black hole with a global monopole," International Journal of Theoretical Physics, vol. 56, no. 7, pp. 2061-2070, 2017.

[35] M. H. Ali and K. Sultana, "Particles' tunneling from cosmic string black holes surrounded by quintessence," International Journal of Theoretical Physics, vol. 56, no. 7, pp. 2279-2292, 2017.

[36] W. Javed, R. Ali, and G. Abbas, "Charged vector particles tunneling from 5D black hole and black ring," 2017, https:// arxiv.org/abs/1705.05702.

[37] W. Javed, G. Abbas, and R. Ali, "Charged vector particle tunneling from a pair of accelerating and rotating and 5D gauged super-gravity black holes," The European Physical Journal C, vol. 77, no. 5, 2017.

[38] K. Jusufi, "Quantum tunneling of spin-1 particles from a 5D Einstein-Yang-Mills-Gauss-Bonnet black hole beyond semiclassical approximation," EPL (Europhysics Letters), vol. 116, no. 6, Article ID 60013, 2016.

[39] K. Jusufi and G. Apostolovska, "Hawking radiation of Dirac monopoles from the global monopole black hole with quantum gravity effects," Astrophysics and Space Science, vol. 361, no. 12, article 374, 2016.

[40] M. H. Ali and K. Sultana, “Charged particles' Hawking radiation via tunneling of both horizons from Reissner-Nordström-TaubNUT black holes," International Journal of Theoretical Physics, vol. 52, no. 8, pp. 2802-2817, 2013.

[41] M. H. Ali, "Charged particles' tunneling from Hot-NUT-KerrNewman-Kasuya spacetime," International Journal of Theoretical Physics, vol. 47, no. 9, pp. 2203-2217, 2008.

[42] M. H. Ali, "Hawking radiation via tunneling from hot NUTKerr-Newman-KASuya spacetime," Classical and Quantum Gravity, vol. 24, no. 23, pp. 5849-5860, 2007.

[43] I. Sakalli, M. Halilsoy, and H. Pasaoglu, "Erratum to: Fading Hawking radiation," Astrophysics and Space Science, vol. 340, no. 1, pp. 155-160, 2012.

[44] I. Sakalli, M. Halilsoy, and H. Pasaoglu, "Entropy conservation of linear dilaton black holes in quantum corrected Hawking radiation," International Journal of Theoretical Physics, vol. 50, no. 10, pp. 3212-3224, 2011.

[45] H. Pasaoglu and I. Sakalli, "Hawking radiation of linear dilaton black holes in various theories," International Journal of Theoretical Physics, vol. 48, no. 12, pp. 3517-3525, 2009.

[46] I. Sakalli and A. Ovgun, "Hawking radiation of spin-1 particles from a three-dimensional rotating hairy black hole," Journal of Experimental and Theoretical Physics, vol. 121, no. 3, pp. 404407, 2015.

[47] K. Nozari and A. S. Sefiedgar, "Comparison of approaches to quantum correction of black hole thermodynamics," Physics Letters B, vol. 635, no. 2-3, pp. 156-160, 2006.

[48] K. Nozari and A. Etemadi, "Minimal length, maximal momentum, and Hilbert space representation of quantum mechanics," Physical Review D: Particles, Fields, Gravitation and Cosmology, vol. 85, Article ID 104029, 2012.

[49] A. Kempf, G. Mangano, and R. B. Mann, "Hilbert space representation of the minimal length uncertainty relation," Physical Review D: Covering Particles, Fields, Gravitation, and Cosmology, vol. 52, article 1108, 1995.

[50] S. Hossenfelder, M. Bleicher, S. Hofmann, J. Ruppert, S. Scherer, and H. Stöcker, "Signatures in the Planck regime," Physics Letters $B$, vol. 575, no. 1-2, pp. 85-99, 2003. 
[51] A. N. Tawfik and E. A. El Dahab, "Corrections to entropy and thermodynamics of charged black hole using generalized uncertainty principle," International Journal of Modern Physics A, vol. 30, no. 9, Article ID 1550030, 2015.

[52] M. A. Anacleto, F. A. Brito, and E. Passos, "Quantum-corrected self-dual black hole entropy in tunneling formalism with GUP," Physics Letters B, vol. 749, pp. 181-186, 2015.

[53] M. Dehghani, "Corrections to the Hawking tunneling radiation in extra dimensions," Physics Letters B, vol. 749, pp. 125-129, 2015.

[54] A. Farag Ali, M. M. Khalil, and E. C. Vagenas, "Minimal Length in quantum gravity and gravitational measurements," EPL (Europhysics Letters), vol. 112, no. 2, Article ID 20005, 2015.

[55] K. Nozari and S. H. Mehdipour, "Gravitational uncertainty and black hole remnants," Modern Physics Letters A, vol. 20, no. 38, pp. 2937-2948, 2005.

[56] Z. W. Feng, S. Z. Yang, H. L. Li, and X. T. Zu, "Constraining the generalized uncertainty principle with the gravitational wave event GW150914," Physics Letters B, vol. 768, pp. 81-85, 2017.

[57] S. K. Rama, "Some consequences of the generalised uncertainty principle: statistical mechanical, cosmological, and varying speed of light," Physics Letters B, vol. 519, no. 1-2, pp. 103-110, 2001.

[58] Y. Heydarzade and F. Darabi, "Black hole solutions surrounded by perfect fluid in Rastall theory," Physics Letters B, vol. 771, pp. 365-373, 2017.

[59] Y. Yang, J. Sun, Y. Guo, Q. Li, J. Huang, and Q. Chang, "Study of $\Upsilon(n S) \longrightarrow B_{c} P$ decays with perturbative QCD approach," Physics Letters B, vol. 751, pp. 171-176, 2015.

[60] D. W. Ebner, "Equations of motion of Glashow-SalamWeinberg theory after spontaneous symmetry breaking," Annalen der Physik, vol. 498, no. 1-2, pp. 119-133, 1986.

[61] E. D. Commins and P. H. Bucksbaum, Weak Interactions of Leptons and Quarks, Cambridge University Press, Cambridge, $\mathrm{Uk}, 1983$.

[62] T. Shivalingaswamy and B. A. Kagali, "Eigenenergies of a relativistic particle in an infinite range linear potential using WKB method," European Journal of Physics Education, vol. 2, no. 2, 2011.

[63] X.-Q. Li and G.-R. Chen, "Massive vector particles tunneling from Kerr and Kerr-Newman black holes," Physics Letters B, vol. 751, pp. 34-38, 2015. 

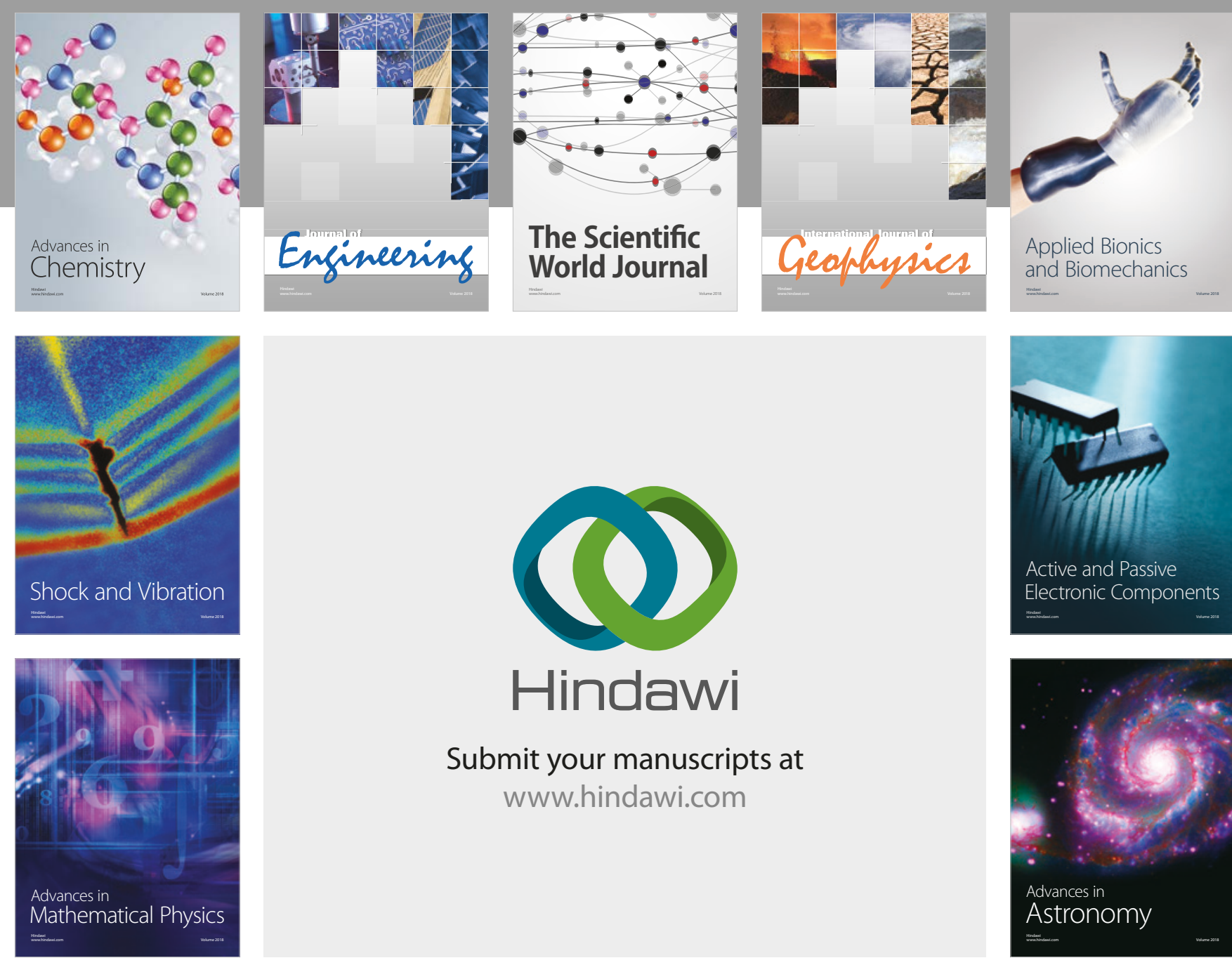

Submit your manuscripts at

www.hindawi.com

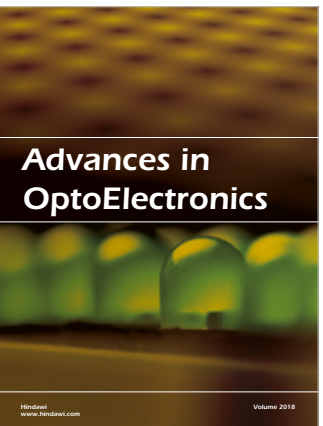

\section{Rotcting Machinery}
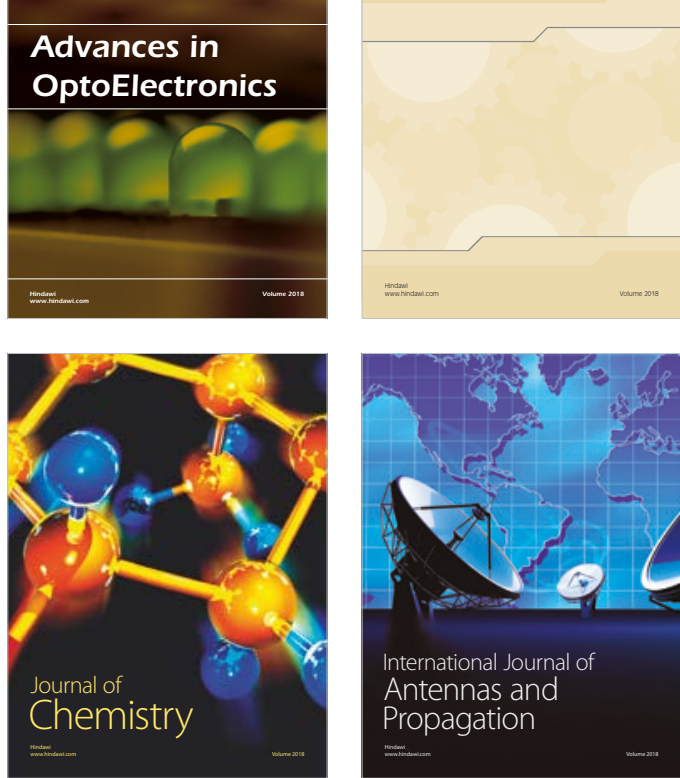

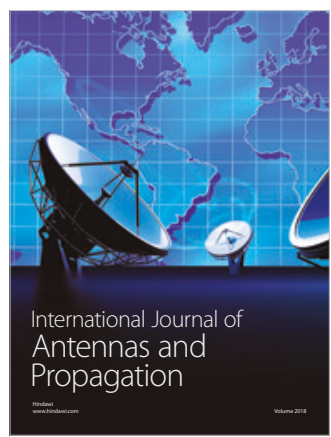

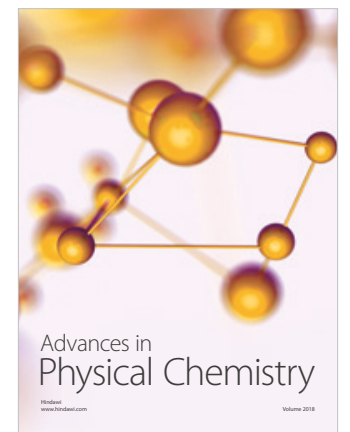

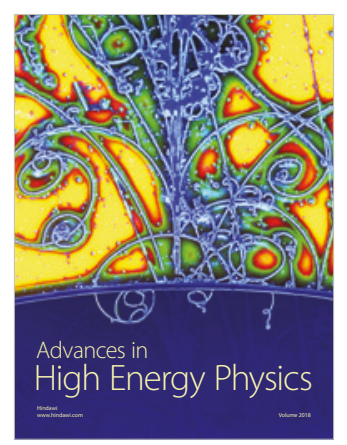

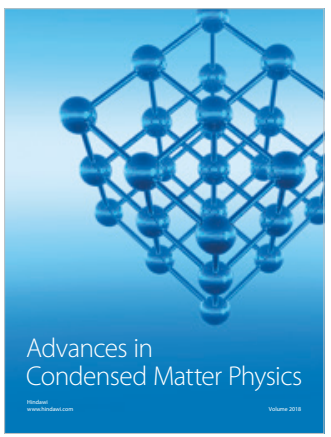

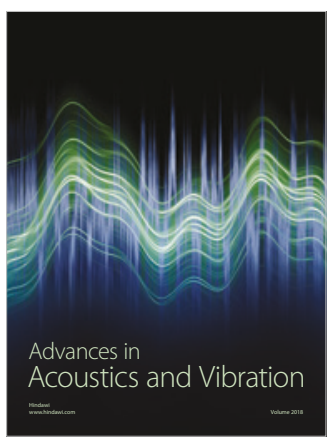

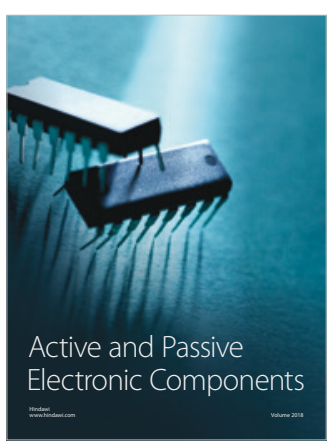
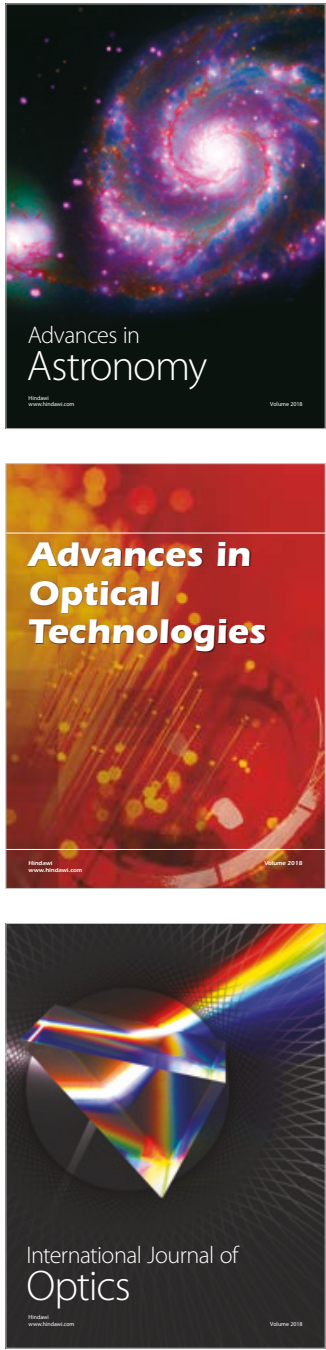